\title{
Life breaks symmetry
}

\author{
Peter Nick ${ }^{1}$
}

Received: 7 June 2016 / Accepted: 7 June 2016 / Published online: 16 June 2016

(C) Springer-Verlag Wien 2016

At the dawn of the twentieth century, Ernst Haeckel's book "Kunstformen der Natur" (Art Forms of Nature, Haeckel 1904) was a major factor to convey to society the ideas of biology in general and evolutionary biology in particular. The aesthetic impression of artistically arranged and depicted life forms inspired enthusiasm for the beauty of nature and can be considered as classical masterpiece of science communication. A good deal of this overwhelming effect is due to the emphasis of symmetry, for instance when Haeckel compiles plates showing diatoms or radiolaria. Symmetry has been a central element of art over the ages, and the celebration of natural symmetry in Haeckel's work served to show nature as artist. But his, admittedly successful, propagandistic trick might be misleading: the beauty of nature is rather opposed to artistic beauty, because the ruling principle is asymmetry, not symmetry. The forced symmetry in the gardens of Versailles symbolises the subjugation of nature by civilisation, and Jacob's famous statement (L'evolution c'est bricolage) of the tinkering evolution (Jacobs 1977) reveals that life has to be asymmetric. There are different arguments for the necessity of biological asymmetry - here, just one will be pursued: to live means to develop, and development requires a deviation from equilibrium. Symmetry (and that may be one reason why it is so attractive for art) represents a state of maximal equilibrium. Real symmetry can only be a static end point, never the start of a developmental process. Life, however, is never in equilibrium; it can only oscillate around the equilibrium. For this

Peter Nick

peter.nick@kit.edu

1 Molecular Cell Biology, Karlsruhe Institute of Technology, Karlsruhe, Germany reason, any developmental process must start with a break of symmetry, and a break of symmetry often heralds a developmental process. Three contributions to the current issue address how life breaks symmetry in plants and animals. The comparison of these cases is revealing.

The egg of the fruitfly Drosophila melanogaster has been a prime model to understand symmetry break in animal development. Here, the maternal follicle imprints anteriorposterior and dorsiventral polarity, for instance by deposition of nontranscribed mRNA encoding the transcription factor BICOID that lays down the anterior pole. The work by Garbiec et al. (2016) investigates follicle development in the neuropteran fly Osmylus fulvicephalus. They find that a specific population of follicle cells, the anterior centripetal cells, extend hypertrophically forming a fold, which sets down a double symmetry break, where not only the anterior, but also the dorsal side is defined. Thus, there are variations to the theme for the model worked out in the fruitfly, although this model is widely used as paradigm for insect embryogenesis in general.

The second example for symmetry comes from plants. Here, the formation of the stomatal apparatus proceeds from a so-called meristemoid, where a differentiated epidermal cell re-enters a stem-cell fate (yielding the guard cell mother cell) and not only generates the guard cells by asymmetric division, but also recruits asymmetric divisions of the neighbouring cell (the subsidiary cell mother cell) to produce subsidiary cells. The presence of a mobile signal indicates a hormonal activity. In fact, a gradient of the phytohormone auxin at the interface between the two mother cells has been observed. The work by Livanos et al. (2016) investigates this phenomenon by different localisation techniques in combination with inhibitor studies. Using a cerium chloride stain (Libik-Konieczny et al. 2014), they can show that hydrogen peroxide accumulates in the same location. They then test the working hypothesis that auxin (which is secreted in a polar fashion) and apoplastic 
oxidative burst might act in concert to set the asymmetry. In fact, they can generate ectopic subsidiary cells by application of exogenous hydrogen peroxide, whereas ROS scavengers or inhibitors of NADPH oxidases interrupt the symmetry break of the subsidiary mother cell. This study indicates that the guard cell mother cell can imprint a symmetry break on the neighbouring cells by polar auxin transport that is than transduced by a gradient of oxidative burst. The question, how this gradient in the abundance of oxidative molecules is transduced into an asymmetric division of the subsidiary cell mother cell promises many interesting research questions that can be asked by investigating this system in the future.

In the two previous examples, a symmetry break was imposed upon immobile cells by their neighbours. What about cells that move? Here, the asymmetry is under continuous challenge, because the morphology is not fixed. The review by Barker et al. (2016) investigates the role of centrioles in non-dividing cells that are often cells on the move. They show that the extracellular signals that drive cellular movement differ, but they can still define the Par proteins and the Rho GTPases as common target. The centrosomal location, in turn, can adjust the localisation of the Golgi apparatus and, through the LINC complexes, of the nucleus. The proper localisation of the Golgi apparatus depends on microtubular motors (both minus-end-directed dyneins and their regulators as well as plus-end-directed kinesins). Despite these common themes, there are (similar to the symmetry break of insect eggs) specific differences between fibroblasts, neural astrocytes or cells of the immune system. These differences seem to be linked with differences in the mechanisms of movement, for instance, whether the movement is driven by a broad lamellipodium as in fibroblasts, or by extension of exploratory processes as in neurites. Despite these specific differences, the symmetry break of mobile animal cells seems to be linked with the dynamic polarity of the microtubules nucleated by the centrosome.

\section{Compliance with ethical standards}

Conflict of interest The author declares that he has no conflict of interest.

\section{References}

Barker A, McIntosh K, Dawe H (2016) Centrosome positioning in nondividing cells. Protoplasma, current issue

Garbiec A, Kubrakiewicz J, Mazurkiewicz-Kania M, Simiczyjew B, Jedrzejowska I (2016) Asymmetry in structure of the eggshell in Osmylus fulvicephalus (Neuroptera: Osmylidae). An exceptional case of breaking symmetry during neuropteran oogenesis. Protoplasma, current issue

Haeckel E (1904) Kunstformen der Natur. Wien, Leipzig

Jacob F (1977) Evolution and tinkering. Science 196:1161-1166

Libik-Konieczny M, Kozieradzka-Kiszkurno M, Desel C, MichalecWarzecha Z, Miszalski Z, Konieczny R (2014) The localization of $\mathrm{NADPH}$ oxidase and reactive oxygen species in in vitro-cultured Mesembryanthemum crystallinum L. Hypocotyls discloses their differing roles in rhizogenesis. Protoplasma 252:477-487

Livanos P, Galatis B, Apostolakos P (2016) Deliberate ROS production and auxin synergistically trigger the asymmetrical division generating the subsidiary cells in Zea mays stomatal complexes. Protoplasma, current issue 